\title{
THE ELECTRONIC TRANSPORT MECHANISM IN AMORPHOUS TETRAHEDRALLY-COORDINATED CARBON FILMS SAVD--97-1673C
}

\author{
J. P. SULLIVAN, T. A. FRIEDMANN, R. G. DUNN, E. B. STECHEL, P. A. SCHULTZ, M.
} P. SIEGAL, and N. MISSERT

Sandia National Laboratories, MS 1421, Albuquerque, NM 87185-1421

CONF-97/201 - -

\section{ABSTRACT}

The electronic transport mechanism in tetrahedrally-coordinated amorphous carbon was investigated using measurements of stress relaxation, thermal evolution of electrical conductivity, and temperature-dependent conductivity measurements. Stress relaxation measurements were used to determine the change in 3-fold coordinated carbon concentration, and the electrical conductivity was correlated to this change. It was found that the conductivity was exponentially proportional to the change in 3-fold concentration, indicating a tunneling or hopping transport mechanism. It was also found that the activation energy for transport decreased with increasing anneal temperature. The decrease in activation energy wa responsible for the observed increase in electrical conductivity. A model is described whereire the transport in this material is described by thermally activated conduction along 3 -fold linkages or chains with variable range and variable orientation hopping. Thermal annealing leads to chain ripening and a reduction in the activation energy for transport.

\section{INTRODUCTION}

Tetrahedrally-coordinated amorphous carbon (ta-C, a-tC, amorphous diamond, amorphic diamond, etc.) is a medium to wide-gap material (about $1 \mathrm{eV}$ to $2.5 \mathrm{eV}$ ) consisting exclusively of carbon (i.e. no hydrogen) with two types of coordination, 4-fold and 3-fold. The band edges have $\pi$ or $\pi^{*}$ character which originate from the 3 -fold coordinated component (the bond hybridization of an ideal 3-fold coordinated atom being three $\mathrm{sp}^{2}$ bonds and one $\pi$ bond). The magnitude of the band gap is determined primarily by the splitting in energy between the $\pi$ and $\pi^{*}$ states which itself is determined by the degree of linking or clustering of the 3 -fold sites. The configuration (whether chain-like, cluster-like, etc.) of the 3 -fold structures embedded in the 4-fold matrix is not known with any certainty. The most energetically favorable configuration is for the 3-fold sites to form compact three-dimensional clusters [1]. However, most recent first principles calculations find that chain-like clustering is the most favored structure with compact (e.g. ring-like) structures being absent [2-4]. This is presumably due to chain-like structures being kinetically easier to form over compact, closed structures.

There are interesting aspects to the electrical properties of ta-C. Despite having a modest band gap, the material does not behave like a conventional semiconductor. The conduction states are localized [5], so the apparent carrier mobilities are quite low. While it is possible to modify the conductivity of these materials by incorporating other elements, e.g. $\mathrm{N}$ [6], the efficiency of these dopants are very low and there is no evidence that it is possible to form pn junctions [7]. The low mobility and inability to create electrical junctions by doping hinders the use of this material in certain device applications, e.g. bi-polar field effect transistors, photocells, etc. A more promising application for this material is as an electron emitter material for vacuum field emission devices, e.g. field emission flat panel displays or vacuum microelectronics.

ta- $\mathrm{C}$ also differs from conventional semiconductors in that the current conduction through these materials is spatially inhomogeneous. The measured through-thickness (i.e. perpendicular to the film plane) conductivity varies over a large range (greater than one order of magnitude) with no systematic trends nor correlation with extrinsic defects (particles, etc.) [8]. Interestingly, spatially resolved electron emission from these materials also shows large spatial variation in emission current which could be related to the heterogeneity in conductivity [9].

In this work we discuss the electronic transport mechanism in ta-C films. We use an unconventional approach wherein we measure the change in electrical conductivity in the ta- $\mathrm{C}$ film with changes in 3-fold carbon concentration. The change in 3-fold carbon concentration 


\section{DISCLAIMER}

This report was prepared as an account of work sponsored by an agency of the United States Government. Neither the United States Government nor any agency thereof, nor any of their employees, makes any warranty, express or implied, or assumes any legal liability or responsibility for the accuracy, completeness, or usefulness of any information, apparatus, product, or process disclosed, or represents that its use would not infringe privately owned rights. Reference herein to any specific commercial product, process, or service by trade name, trademark, manufacturer, or otherwise does not necessarily constitute or imply its endorsement, recommendation, or favoring by the United States Government or any agency thereof. The views and opinions of authors expressed herein do not necessarily state or reflect those of the United States Government or any agency thereof. 
itself is determined by modeling the stress relaxation which occurs in these materials as a result of thermal annealing. The advantage of this approach is that it leads to an unambiguous interpretation of the conduction mechanism, and it permits possible identification of the structural changes which occur in the material following thermal annealing.

\section{EXPERIMENTAL}

The ta-C films used in this study were prepared using pulsed-laser deposition with a $\mathrm{KrF}$ laser and a graphite target. Deposition was performed in vacuum (better than $1 \times 10^{-6}$ Torr) up to a film thickness of 100 to $120 \mathrm{~nm}$ using a laser fluence of $\geq 50 \mathrm{~J} / \mathrm{cm}^{2}$. These deposition conditions led to films with density of $\sim 3.0 \mathrm{~g} / \mathrm{cm}^{3}$, as measured by $\mathrm{x}$-ray reflectivity, corresponding to about 70\% 4-fold and 30\% 3-fold carbon. For stress relaxation measurements, the films were deposited upon 2" Si wafers which were treated prior to deposition by brief immersion in diluted HF. For electrical measurements, the films were deposited upon a layer of TiW (200 nm thick) on $\mathrm{SiO}_{2}(1 \mu \mathrm{m})$ on $\mathrm{Si}$.

Thermal annealing was performed ex situ in a rapid thermal annealer in an ambient of flowing Ar. Anneals at successively longer time intervals at the same temperature were performed in order to determine time-dependent changes. The film stress was measured at room temperature following annealing by examining wafer curvature and using Stoney's equation. Contacts ( $\mathrm{Ti}$, followed by $\mathrm{Au}$ ) were photolithographically patterned on to the ta-C film surface and had areas ranging from $2.5 \times 10^{-5} \mathrm{~cm}^{-2}$ to $1.6 \times 10^{-3} \mathrm{~cm}^{-2}$. The DC electrical conductivity of the samples was determined by measuring current-voltage characteristics between a top contact and the TiW layer beneath the ta-C film. A bias range of $-50 \mathrm{mV}$ to $+50 \mathrm{mV}$ was used. Ohmic conduction was observed within this range. Measurement of the activation energies for electrical transport were made by performing temperature-dependent conductivity measurements over the range $230 \mathrm{~K}$ to $380 \mathrm{~K}$. No irreversible changes in electrical conductivity of the samples were observed following cycling through this temperature range.

\section{RESULTS AND DISCUSSION}

Fig. 1(a) shows the measured film stress and normalized conductivity in ta-C films following time-temperature annealing. The film stress drops continuously with annealing from a value of about $7 \mathrm{GPa}$ (compressive) in the as-deposited state to zero after annealing at $600^{\circ} \mathrm{C}$. Most film properties show little or no change following complete stress relaxation at this temperature [10]. The conductivity, Fig. 1(b), does show pronounced change, however. Prior to annealing, the conductivity showed considerable variation from contact to contact, but the median value was $\sim 1 \times 10^{-7}(\Omega \mathrm{cm})^{-1}$. Following thermal annealing, the conductivity continued to show large variation from site to site, but after normalization to the initial conductivity, $\sigma_{\text {init, }}$ approximate universal behavior was observed for all contacts. The data shown in Fig. 1(b) is for contacts that exhibited median behavior.

We have developed a model to explain the stress relaxation which occurs in ta- $\mathrm{C}$ following thermal annealing [8]. The model assumes that thermal annealing causes some 4-fold coordinated carbon atoms to convert to 3-fold coordination with first order kinetics and a classical activation barrier, $E_{A}$ [11]. The stress relaxation arises from the fact that 3-fold coordinated carbon atoms have, on average, shorter bond lengths than 4-fold coordinated atoms (an $\mathrm{sp}^{2}$ bond is about $8 \%$ shorter than an $\mathrm{sp}^{3}$ bond). The $\pi$ bond of the 3 -fold atom is much longer, however, so that the average volume occupied by a 3-fold atom is larger than that of a 4fold atom (i.e. conversion leads to a drop in film density [12]). Because of this difference in $\mathrm{sp}^{2}$ and $\mathrm{sp}^{3}$ bond length, strain relief is possible if the newly created 3 -fold atoms are oriented preferentially with the shorter $\mathrm{sp}^{2}$ bonds in the plane of the film. The driving force for this preferential orientation is strain energy: due to the biaxial stress state of the film, there is a reduction in strain energy when 3-fold sites are created with the preferential orientation while non-optimal orientations, e.g. with the $\pi$ bond in the film plane, cause an increase in strain energy. Finally, because the films are amorphous, there is a distribution of activation energies, $E_{A}$, needed to convert 4-fold to 3-fold. In its simplest form, the model time-temperature stress relaxation response is given by 

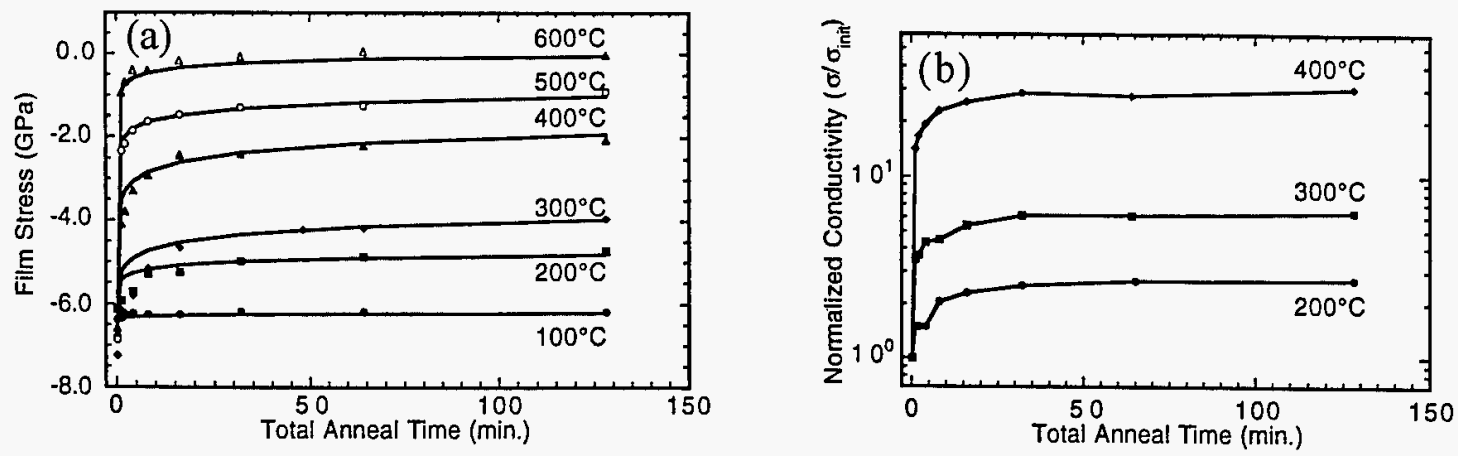

Fig. 1. (a) Time and temperature dependence of the stress relaxation in ta-C. Solid lines are fits to the data using the model in the text. (b) Time and temperature dependence of the conductivity change in ta-C. Solid lines are guides.

$$
\sigma(t, T)=\sigma_{0}+\frac{\varepsilon_{s p^{2}-s p^{3}} E^{\infty}}{1-v} \int_{0}^{\infty} N\left(E_{A}\right)\left\{1-\exp \left[-v_{0} t \exp \left(-E_{A} / k_{B} T\right)\right]\right\} d E_{A},
$$

where $\sigma(\mathrm{t}, \mathrm{T})$ is the time and temperature-dependent stress, $\sigma_{0}$ is the initial in-plane stress, $\mathrm{E}$ is the elastic modulus of ta-C, $\sim 1000 \mathrm{GPa}$ [13], $v$ is the Poisson ratio, $\sim 0.1, \varepsilon_{s p^{2}-s^{3}}$ is the strain associated with replacing a 4-fold atom with a 3-fold atom $(\sim-0.08), v_{0}$ is the attempt frequency (about $10^{13} \mathrm{sec}^{-1}$ ), and $\mathrm{N}\left(\mathrm{E}_{\mathrm{A}}\right) \mathrm{dE}_{\mathrm{A}}$ is the fraction of 3 -fold sites converted from 4 -fold sites with an activation energy in the range of $\mathrm{dE}_{\mathrm{A}}$.

Fitting Eq. 1 to the stress relaxation data of Fig. 1(a), the distribution of activation energies, $\mathrm{N}\left(\mathrm{E}_{\mathrm{A}}\right)$ was obtained. The activation energies were found to lie in the range of $<1 \mathrm{eV}$ to over 3 $\mathrm{eV}$, with a peak near $2 \mathrm{eV}$. Recently, Reznik et al. found an activation energy of $0.7 \mathrm{eV}$ for conversion of $\mathrm{sp}^{3}$ bonds to $\mathrm{sp}^{2}$ bonds at defect sites in crystalline diamond, which is near the low end of the range observed here [14]. With the measured $N\left(E_{A}\right)$, the change in 3-fold concentration, $\Delta \mathrm{C}_{3}$-fold, can be determined (equal to the integral in Eq. 1). For full stress relaxation, only a small increase in 3-fold concentration of about 6.5 at. \% is required, i.e. for an initial 3-fold concentration of $30 \%$, the concentration following stress relief may increase to as little as $36.5 \%$.

The derived change in 3-fold concentration can be combined with the conductivity data in Fig. 1 to obtain a correlation between electrical conductivity and 3-fold concentration, Fig. 2. An approximately linear relationship was obtained, indicating that

$$
\sigma=\sigma_{\text {init }} \exp \left(\kappa \Delta C_{3-\text { fold }}\right),
$$

where $\kappa \approx 100$. The temperature-dependent conductivity was also measured for several contacts as a function of annealing temperature. Over the temperature range investigated in this study, linear plots were obtained for both $\log$ (conductivity) vs. $1 / \mathrm{T}$ or $\log$ (conductivity) vs $1 / \mathrm{T} \alpha$, where $\alpha=1 / 4$, so we can not unambiguously determine the hopping conduction mechanism alone based on temperature-dependent conductivity measurements [15]. Using plots of $\log$ (conductivity) vs. $1 / \mathrm{T}$, we obtain "activation

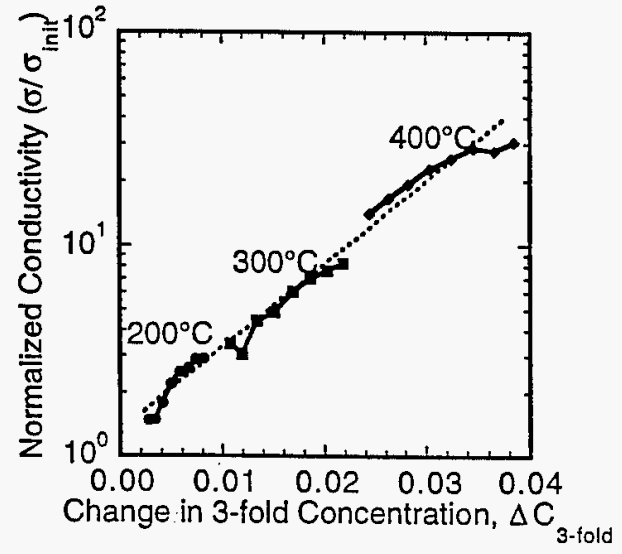

Fig. 2. Electrical conductivity in ta-C as a function of change in 3-fold concentration. The temperatures on the plot indicate the temp. at which the sample was annealed. 

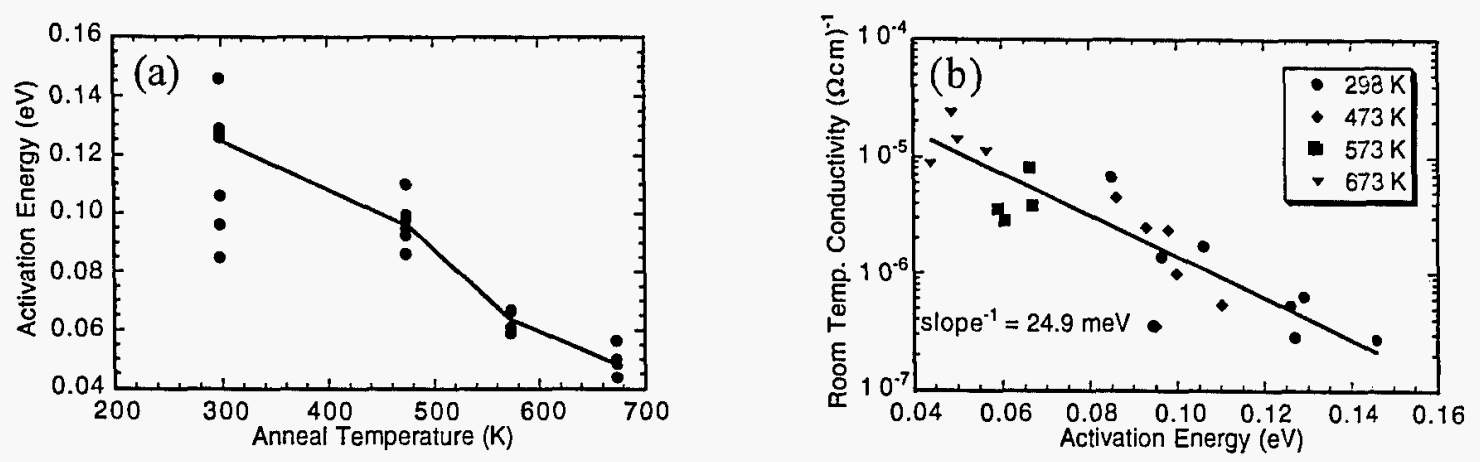

Fig. 3. (a) Activation energy for transport for ta-C films annealed at different temperatures. Line is drawn through the median values. (b) Room temperature conductivity as a function of activation energy for annealed and unannealed ta-C films. Solid line is a fit to the data.

energies" for electronic transport, $E_{A}$, and these are shown in Fig. 3(a). Annealing was found to systematically lower the activation energy for transport. Furthermore, the drop in activation energy is sufficient to fully explain the increase in electrical conductivity following annealing, as is shown in Fig. 3(b).

In Fig. 3(b), the room temperature conductivity is plotted as a function of the measured activation energy for that particular contact. Data for all contacts and for all annealing temperatures are included. Fitting a line through the data, an inverse slope equal to $24.9 \mathrm{meV}$ was obtained. This is close to the value of $\mathrm{kB}_{\mathrm{B}} \mathrm{T}$ at room temperature, which indicates that to good approximation, the conductivity for a particular contact, $i, \sigma_{i}$, is given by $\sigma_{i}=\sigma_{0} \exp \left(-E_{A, i} / k_{B} T\right)$, where $\sigma_{0}$ is the pre-exponential factor for electrical transport and is constant, $\approx 9 \times 10^{-5}(\Omega \mathrm{cm})^{-1}$, and $\mathrm{E}_{\mathrm{A}, \mathrm{i}}$ is the activation energy for contact $\mathrm{i}$.

Summarizing these results, (1) the measured electrical conductivity of ta- $\mathrm{C}$ films is exponentially proportional to changes in 3-fold concentration, $\Delta \mathrm{C}_{3}$-fold, (2) the activation energies for electrical transport are small, but the pre-exponential factors are very small, so the conductivity is low, (3) annealing leads to a systematic reduction in the activation energy for electrical transport, and (4) these changes in apparent activation energy are sufficient to explain the change in conductivity (i.e. the pre-exponential factor does not change). From (1) and (2) it can be concluded that the current conduction mechanism is that of hopping transport [15]. Within the mechanism of hopping conductivity, several possibilities exist for how annealing may change the electrical conductivity: (i) increasing $\Delta \mathrm{C}_{3}$-fold may increase the number of hopping sites, effectively reducing the mean hopping distance, (ii) increasing $\Delta \mathrm{C}_{3 \text {-fold }}$ may lead to an increase in the size of the 3-fold clusters or linkages (e.g. ripening) which would also decrease the hopping distance, or (iii) increasing $\Delta \mathrm{C}_{3 \text {-fold }}$ may lower the activation energy for transport, and this leads to the enhanced conduction. From (3) and (4), above, we can conclude that (i) and (ii) are not the major contributors to the conductivity change with annealing and that the most important effect is (iii).

The reduction in activation energy for electrical transport with thermal annealing may arise from ripening of the 3-fold clusters or linkages. Within the vicinity of a 3-fold cluster or linkage, the local DOS shows an energy gap equal to the energy splitting between the $\pi$ and $\pi^{*}$ states, $E_{\pi-\pi^{*}}$. The magnitude of $E_{\pi-\pi^{*}}$ is a function of the number of carbon atoms in the 3 -fold cluster or linkage. For isolated 3 -fold chains, Robertson has shown that $\mathrm{E}_{\pi-\pi^{*}}$ varies as $\sim 20 \mathrm{eV} / \mathrm{N}$ where $\mathrm{N}$ is the number of carbon atoms in the chain; a slightly different dependence is obtained if the carbon atoms make compact ring clusters [1]. This dependence of $E_{\pi-\pi^{*}}$ on $N$ is shown in Fig. 4. In Fig. 4 we also include two data points for recent results of Frauenheim et al. which involve a 6-member and an 8-member 3-fold carbon chain embedded in a 4-fold amorphous carbon matrix, i.e. the calculated structure of ta-C [3]. These two points lie very close to the 
values calculated by Robertson for isolated chains.

If we assume that carriers are thermally activated into the $\pi$ or $\pi^{*}$ states of chain-like linkages of 3-fold atoms, then thermal activation will be easiest (the barrier smallest) for the longest chains (these have the smallest $\mathrm{E}_{\pi-\pi^{*} \text { ). }}$. Hopping conduction therefore constitutes tunneling from long chain to long chain with the activation energy for transport reflecting both the activation energy for transport along the chain (i.e. thermal activation to an extended state of the chain plus the activation energy for propagation along the chain [16]) as well as the activation energy needed to hop between chains having $\pi$ or $\pi^{*}$ states at different energies. The measured low values for the activation energies suggests that the Fermi level in ta-C likely lies near the $\pi$ states of the longest chains, which is consistent with the reported $\mathrm{p}$-type character of undoped ta-C [6].

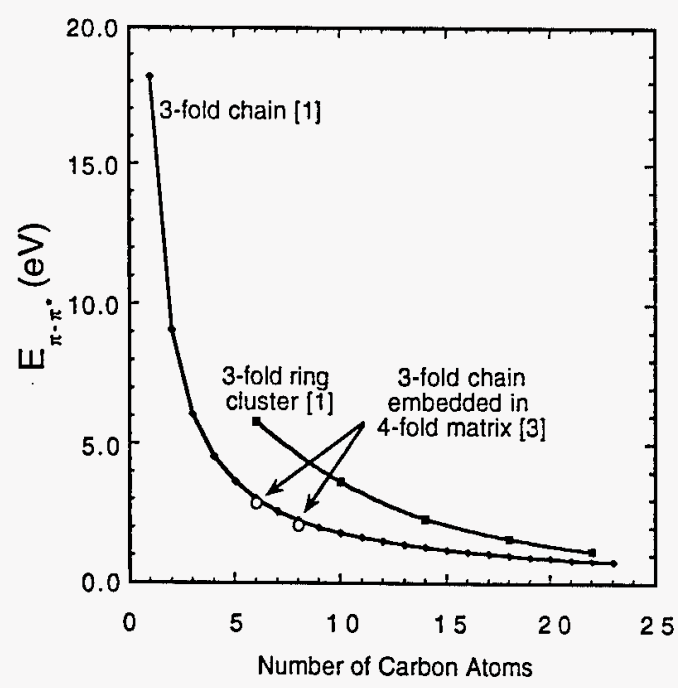

The view of the hopping conductivity that emerges is as follows: (i) carriers are preferentially activated into the longest 3 -fold chains or linkages, (ii) carriers tunnel from long chain to long chain (intervening 3-fold chains have larger $\mathrm{E}_{\pi-\pi^{*}}$, and hence their $\pi$ and $\pi^{*}$ states do not overlap with the $\pi$ and $\pi^{*}$ states of the long chains), but it is also necessary that the $\pi$ states of the two long chains have similar orientation (the tunnel probability for orthogonal orientations drops to zero), (iii) thermal annealing causes chain ripening, and (iv) as chains lengthen, $E_{\pi-\pi^{*}}$ decreases and the activation energy for transport decreases. The hopping conductivity can be thought of as being both variable range and "variable orientation" - this last point referring to the orientational disorder between the $\pi$ conduction states. This disorder is an important part of the conduction process; it has been shown that disorder in the $\pi$ orbital orientation is one of the primary reasons for the strong localization in ta-C [5]. It should also be noted that the conduction process discussed here is considerably different from the nearest neighbor hopping conduction in a-C proposed by Dasgupta et al. [17].

An implication of this hopping conduction process is that the current conduction process will be highly heterogeneous. Consider a random resistor network model for ta-C. The lowest resistance connections between nodes will be those for which two long chains have similar $\pi$ orbital orientations and are in close proximity. These will be relatively rare (optical absorption measurements suggest that only very few large 3 -fold linkages can exist). The optimal current pathways must link up several of these rare low resistance pathways in order to propagate current completely through the film, which is the reason that large spatial variation in conductivity may be observed.

Finally, given the measured relationship between conductivity, $\Delta C_{3 \text {-fold, and }} E_{A}$ (Figs. 2 and 3), it is possible to determine an estimate for a typical size of a 3 -fold chain or linkage responsible for current conduction [18]:

$$
N=\beta^{\prime} / \kappa C_{0} k_{B} T,
$$

where $\mathrm{N}$ is the number of carbon atoms in the chain or linkage, $\beta^{\prime}$ is the relationship between activation energy and $\mathrm{N}, \kappa$ is from Eq. 2 , and $\mathrm{C}_{0}$ is the initial carbon 3 -fold concentration (prior to annealing). Using $\beta^{\prime}=0.5 \mathrm{E}_{\pi-\pi^{*}}=10 \mathrm{eV} / \mathrm{N}, \kappa=100, \mathrm{C}_{0}=0.3$, and $\mathrm{k}_{\mathrm{B}} \mathrm{T}=0.025 \mathrm{eV}$, then we obtain $\mathrm{N} \approx 13$ carbon atoms. It can be shown that thermal annealing at $400^{\circ} \mathrm{C}$ need only increase $\mathrm{N}$ from 13 to 14 (i.e. ripening of only one atom), and this would be sufficient to account for the approximate 35 times increase in electrical conductivity. 


\section{CONCLUSIONS}

The nature of the electrical transport mechanism in tetrahedrally-coordinated amorphous carbon films was investigated by studying the dependence of the electrical conductivity on 3fold carbon concentration and the change in conductivity and transport activation energy following thermal annealing. An exponential dependence of the electrical conductivity on changes in carbon 3-fold content was found, indicating a hopping conduction process. The activation energy for transport was found to decrease with annealing temperature, suggesting ripening of 3 -fold linkages. The conduction behavior is best described by thermally activated conduction along 3-fold chains or linkages combined with variable range and variable orientation tunneling, primarily between the longest chains or linkages. This conduction process leads to heterogeneity in the current conduction pathways through the sample.

\section{ACKNOWLEDGMENTS}

Contributions by D. Tallant and A. Baca are gratefully acknowledged. This work was supported by the U.S. DOE under Contract DE-AC04-94AL85000. Sandia is a multiprogram laboratory operated by Sandia Corp., a Lockheed Martin Co., for the U.S. DOE.

\section{REFERENCES}

1. J. Robertson, Adv. Phys. 35, 317 (1986).

2. N. A. Marks, D. R. McKenzie, B. A. Pailthorpe, M. Bernasconi, and M. Parrinello, Phys. Rev. Lett. 76, 768 (1996).

3. Th. Frauenheim, G. Jungnickel, Th. Köhler, P. Stitch, and P. Blaudeck, Proc. 1st Int. Meeting on Amorphous Carbon (World Scientific Publ., Singapore, 1998).

4. $\quad$ P. A. Schultz and E. B. Stechel, to appear in Phys. Rev. B, 1998.

5. J. Robertson, Phil. Mag. B 76, 335 (1997).

6. V. S. Veerasamy, J. Yuan, G. A. J. Amaratunga, W. I. Milne, K. W. R. Gilkes, M. Weiler, and L. M. Brown, Phys. Rev. B 48, 17954 (1993).

7. C. Ronning, U. Griesmeier, M. Gross, H. C. Hofsäss, R. G. Downing, and G. P. Lamaze, Diamond Relat. Mater. 4, 666 (1995).

8. J. P. Sullivan, T. A. Friedmann, and A. G. Baca, J. Electron. Mater. 26, 1021 (1997).

9. N. Missert, T. A. Friedmann, J. P. Sullivan, and R. G. Copeland, Appl. Phys. Lett. 70, 1995 (1997).

10. J. P. Sullivan, T. A. Friedmann, D. R. Tallant, J. Mikkalson, D. Rieger, A. G. Baca, and L. J. Martiñez-Miranda, submitted to Appl. Phys. Lett., 1996.

11. We may also consider $E_{A}$ to be an activation energy to re-orient an existing 3-fold site, but since the observed drop in density following annealing is consistent with increasing the 3-fold concentration, we will focus on a conversion mechanism.

12. L. J. Martiñez-Miranda, J. P. Sullivan, T. A. Friedmann, M. P. Siegal, and N. J. DiNardo, Mat. Res. Soc. Proc., Vol. 498, 1998.

13. T. A. Friedmann, J. P. Sullivan, J. A. Knapp, D. R. Tallant, D. M. Follstaedt, D. L. Medlin, and P. B. Mirkarimi, Appl. Phys. Lett. 71, 3820 (1997).

14. A. Reznik, V. Richter, and R. Kalish, Phys. Rev. B 56, 7930 (1997).

15. N. F. Mott and E. A. Davis, Electronic Processes in Non-Crvstalline Materials (Clarendon, Oxford, 1971).

16. If phonon modes were soft along the chains, then carriers could self trap within the chain, and there would be an activation energy for polaronic conduction.

17. D. Dasgupta, F. Demichelis, and A. Tafliaferro, Phil. Mag. B 63, 1255 (1991).

18. J. P. Sullivan and T. A. Friedmann, Proc. 1st Int. Meeting on Amorphous Carbon (World Scientific Publ., Singapore, 1998). 
M98003136

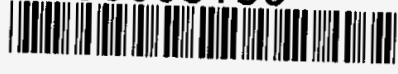

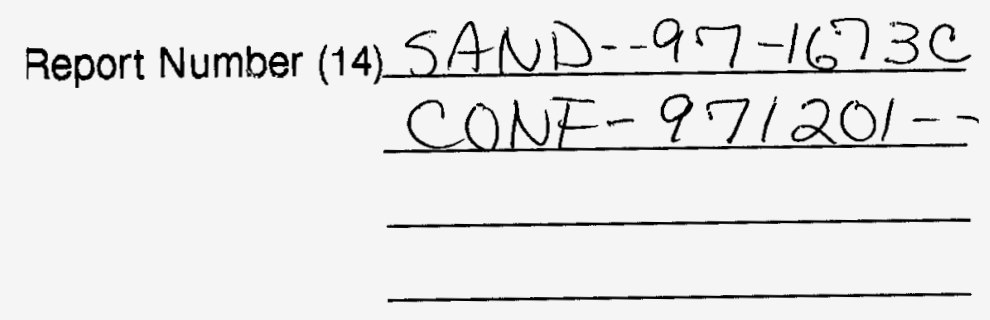

Dubl. Date (11) 199802 sponsor Code (18)
sc Category (19)

DOE 